American Medical Journal 1 (2): 151-156, 2010

ISSN 1949-0070

(C) 2010 Science Publications

\title{
Identification of Novel Human Immunodeficiency Virus-1 Integrase Inhibitors by Shape-Based Virtual Screening
}

\author{
T.K. Omprakash, A. Thamarai Selvan, A. Shahul Hameed and S.P. Geetha \\ Department of Chemistry, Thiagarajar College, Madurai-625009, Tamilnadu, India
}

\begin{abstract}
Problem statement: Human Immunodeficiency Virus-1 (HIV-1) is causative agent of the immune system disease, Acquired Immune Deficiency Syndrome (AIDS). Majority of anti-HIV drugs target reverse transcriptase and protease enzymes. Toxicity and development of multidrug resistant HIV-1 virus strains are the reasons for studying new targets in HIV-1 replication process for identifying novel inhibitor with low toxicity and high activity. Approach: In this study, ROCS software was used to identify the novel HIV-1 Integrase (HIV-1 IN) inhibitor by shape-based virtual screening. The currently used drug raltegravir was used as query molecule. Results: Here five novel molecules were identified, among them Rank 5 molecule was shown to have higher structural and electrostatic similarity to query molecule and this molecule was considered as good inhibitor of HIV-1 IN enzyme. Conclusion: ROCS, EON and FRED effectively identified one active inhibitor of HIV-1 IN among five compounds, which was similar to the query molecule and this study showed that ROCS, EON and FRED can play a vital role in drug discovery projects.
\end{abstract}

Key words: HAART, human immunodeficiency, integrase inhibitors, virtual screening, ROCS

\section{INTRODUCTION}

The immune system disease, Acquired Immune Deficiency Syndrome (AIDS) is caused by Human Immunodeficiency Virus-1 (HIV-1), which belongs to Retrovirus family, which means RNA is genetic material for HIV-1. The size of HIV-1 is approximately 60 times smaller than a RBC and roughly spherical in shape and about $120 \mathrm{~nm}$ in diameter. Though it is a retrovirus, its shape is entirely different from other retroviruses. HIV-1 genome encodes three enzymes namely Reverse Transcriptase, Integrase and Protease which are essential for HIV-1 replication. The majority of anti-HIV drugs target Reverse Transcriptase and Protease enzymes. There are four different classes of drugs available to suppress the viral load, namely Nucleoside Reverse Transcriptase Inhibitors (NRTIs (e.g., AZT, DDI, DDC)), Non-Nucleoside Reverse Transcriptase Inhibitors (NNRTI's (e.g., Etravirine, Nevirapine)), Protease Inhibitors (PIs (e.g., Saquinavir, Lopinavir)) and fusion inhibitors (enfuvirtide). Highly Active Antiretroviral Therapy (HAART) combatively suppresses HIV-1 replication and progression of HIV-1 infection which uses combination of three or more drugs from at least two different classes approved by US Food and Drug Administration (FDA). Patients on long-term HAART medication were affected from development of multidrug resistant HIV-1 virus strains and severe side effects which are the reasons for studying new targets in HIV-1 replication process for discovering safe and potent drugs (Potter et al., 2003; Yusa and Harada, 2004; Fontas et al., 2004; De Bethune and Hertogs, 2006; Potter et al., 2006; Machouf et al., 2006; Tozzi et al., 2006; Cozzi-Lepri, 2007).

HIV-1 Integrase (IN) is an effective target for antiretroviral therapy which inhibit the HIV-1 replication (Thomas and Brandy, 1997). The mechanism of integration of viral DNA into host DNA is carried out by HIV-1 IN enzyme (Asante-Appiah and Skalka, 1997; Hindmarsh and Leis, 1999). For this integration mechanism, the following two steps are essential. In the first step, two deoxynucleotides are removed from the 3'-ends of the viral DNA which is called 3'-end processing and the Second step, in which the processed 3'-ends of the viral DNA (target DNA) are covalently bind to the host chromosomal DNA is called strand transfer reaction. For both of the reactions (3'-end processing and Strand transfer) and for the formation of viral DNA-IN complex, divalent metal cations such as $\mathrm{Mg}^{2+}$ or $\mathrm{Mn}^{2+}$ are required (Ellison and Brown, 1994; Wolfe et al., 1996). HIV-1 IN is a $32 \mathrm{kDa}$ enzyme that consists of three functional domains (Andrake and Skalka, 1996). (i) N-terminal domain stabilized by $\mathrm{Zn}^{2+}$ which binds to HHCC motif ("Zinc finger"-like motif) (Lee and Han, 1996; Zheng et al.,

Corresponding Author: T.K. Omprakash, Department of Chemistry, Thiagarajar College, Madurai-625009, Tamilnadu, India 
1996; Lee et al., 1997) and this is also responsible for multimerization (Zheng et al., 1996). (ii) The catalytic domain consists of conserved D, D-35-E motif, which is represented in IN by the residues ASP 64, ASP 116 and Glu 152 and these residues are play a vital role for catalytic activity and are also coordinated by two metal ion cofactors (Ellison and Brown, 1994; Wolfe et al., 1996; Vink et al., 1994; Hazuda et al., 1997; Beese and Steitz, 1991) and (iii) C-terminal domain has DNAbinding activity which is non-specific (Woerner and Marcus-Sekura, 1993; Engelmen et al., 1994). Using Nuclear Magnetic Resonance (NMR) technique, the structure of N-terminal and C-terminal domain of HIV1 IN was determined (Lodi et al., 1995; Cai et al., 1997; Eijkelenboom et al., 1995); on the other hand catalytic domain of HIV-1 IN was resolved by X-ray crystallography (Dyna et al., 1994; Bujacz et al., 1995).

Figure 1 shows some promising inhibitors of the HIV-1 IN (Cocohoba and Dong, 2008; Hazuda et al., 2000; Di Santo et al., 2005; Shiomi et al., 2005) and some natural peptides are also reported as inhibitor of HIV-1 IN (Marchand et al., 2006; Robinson et al., 1998; Krajewski et al., 2004). Raltegravir is the first HIV-1 IN inhibitor to be approved by the US FDA for antiretroviral treatment (Cocohoba and Dong, 2008) and it is active against multidrug resistant HIV-1 viruses (Morales-Ramirez et al., 2005; Summa et al., 2006; Laufer et al., 2006; Markowitz et al., 2006). Some computational studies on HIV-1 IN inhibitors have been published (Yuan and Parrill, 2002; Makhija and Kulkarni, 2001; 2002). The research reported in this article attempts to design novel HIV-1 IN inhibitors by shape-based virtual screening. In this method there is no need for any structural information of target protein and some researches on shape-based virtual screening were useful in this study (Rush et al., 2005; Gundersen et al., 2005; Muchmore et al., 2006).

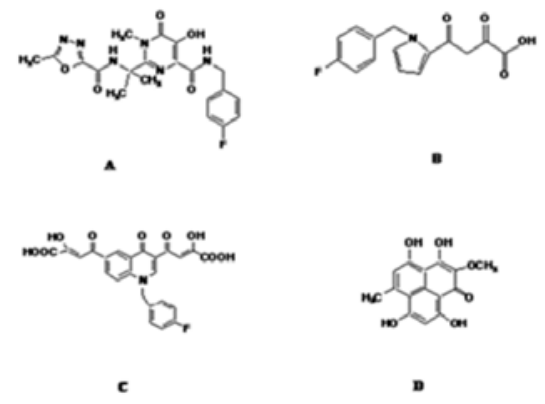

Fig. 1: Some promising inhibitor of HIV integrase, (A) Raltegravir (Cocohoba and Dong, 2008), (B) Diketo Acids (DKA) (Hazuda et al., 2000), (C) Quinolin-4-one derivatives (Di Santo et al., 2005) and (D) natural products (Shiomi et al., 2005)

\section{MATERIALS AND METHODS}

Database preparation: Highly reliably commercial drug-like database was downloaded from ZINC website (John and Brain 2005). This database contains $>3000$ molecules. The drug-like molecules are present in the database as SMILES format. Then these molecules are expanded into a set of 3D conformers by OMEGA software with $0.8 \mathrm{rms}$ and erange $5 \mathrm{kcal} \mathrm{mol}^{-1}$. OMEGA generates different conformations of the ligands (OMEGA ver.2.1.0, 2006) and has been found to be successful in creating bioactive conformations (Bostrom et al., 2003). In OMEGA connection table method is used for generating initial set of 3D coordinates of the given molecule. Merck molecular force field is used to refine the molecules (Halgren, 1996). The Quacpac software is used to assign the AM1-BCC charges (QuacPac ver.1.3, 2006).

Virtual screening: Rapid Overlay of Chemical Structures (ROCS) software is used for to screening the database which contains >30000 conformers. Raltegravir was used as query molecule to screen this database. The query file contains different conformers of the Raltegravir and this approach is called multiconformer query and Fig. 2 shows different conformers of Raltegravir generated by OMEGA. Shape-based superimposition method is used in ROCS. The shape-based virtual screening allows the screening of novel HIV-1 IN inhibitors that are similar in shape with that of the query molecule. In this method there is no need for any structural information of the targets (enzymes and proteins). To maximize the volume overlap between the molecules (query and database molecules), solid-body optimization process is used when molecules are aligned and to compute the geometric overlap atom-centered Gaussian model is used. Shape Tanimoto values were used in this study. Based on ST values, similarities between the two molecules (query and database molecule) were studied and this value ranges from $0-1 ; 1$ for similarity and 0 for dissimilarity. Finally we got three sets of hits and each hit contains best 100 molecules ranked by ST values. Figure 3 shows the query molecule overlapped with database molecules.

Hits from ROCS were used as input to EON which calculates the electrostatic similarity between the query and the database (here ROCS hits are considered as a database) (ROCS ver.2.2 and EON ver.1.1, 2006). Poisson-Boltzmann function is used to calculate the electrostatic similarity and it is measured by Electrostatic Tanimoto (ET) values. This method uses an outer dielectric of 80 . Spin $90^{\circ}$ command was set to maximize electrostatic similarity by spin terminal rotors. 


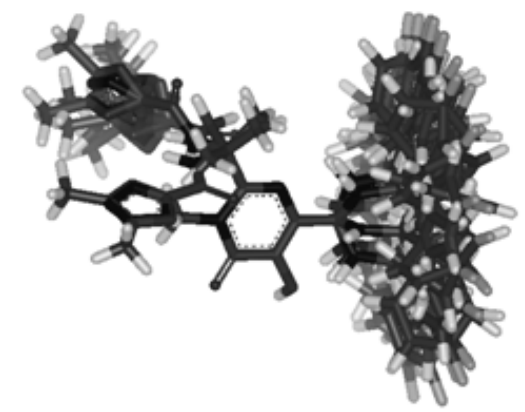

Fig. 2: Different conformers of Raltegravir generated by OMEGA

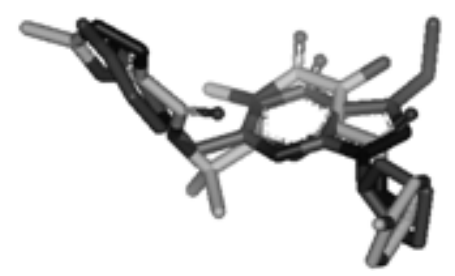

Fig. 3: A rank 5 molecule in Set 1 compounds overlapped with query molecule

Molecular docking: In order to study the binding mode of screened molecules to HIV-1 IN enzyme (PDB id: 1QS4), docking studies were performed. FRED was used to perform docking studies (FRED ver.2.2, 2006). Conformational orientations that clash with the protein or those which are distant from the active sites were rejected (Schulz-Gasch and Stahl, 2003). The default step sizes were 1 and $1.5 \AA$ for translational and rotational modes respectively, which roughly give a Root Mean Square Deviation (RMSD) of 1 for the change. To calculate the docking energies, Chemscore, Shapegauss and PLP scoring functions in FRED were selected.

\section{RESULTS}

A shape-based virtual screening was carried out using ROCS software. Raltegravir was used as query to screen the database for identifying the novel leads which inhibit the HIV-1 IN enzyme. There are two types of methods available for screening, namely single conformer query method and multiconformer query method. In case of multiconformer method, we get the hits for each and every conformer. For example, if multiconformer query file contains 10 conformers of the same molecule, then we get 10 hits and every hit contains different screened molecule. In this study, we have generated 106 conformers for Raltegravir

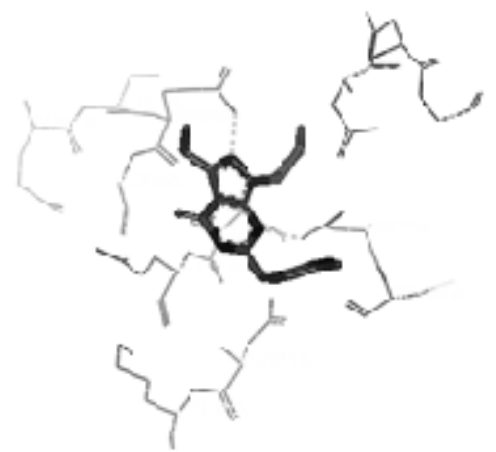

Fig. 4: A rank 5 molecule in set 1 compounds docked with active site of the HIV-1 integrase

Table 1: Hits obtained from rank 1 conformer used as a query

\begin{tabular}{llll}
\hline Rank & ROCS hits & Shape tanimoto & Electrostatic tanimoto \\
\hline 1 & ZINC00017019 & 0.736 & 0.004 \\
2 & ZINC00018810 & 0.726 & 0.007 \\
3 & ZINC0006117 & 0.720 & 0.004 \\
4 & ZINC00023049 & 0.718 & 0.163 \\
5 & ZINC0006708 & 0.711 & 0.510 \\
\hline
\end{tabular}

Table 2: Hits obtained from rank 2 conformer used as a query

\begin{tabular}{llll}
\hline Rank & ROCS hits & Shape tanimoto & Electrostatic tanimoto \\
\hline 1 & ZINC00023931 & 0.730 & -0.011 \\
2 & ZINC00021206 & 0.724 & -0.049 \\
3 & ZINC00023049 & 0.720 & -0.059 \\
4 & ZINC0002303 & 0.718 & 0.006 \\
5 & ZINC00021208 & 0.714 & -0.020 \\
\hline
\end{tabular}

Table 3: Hits obtained from rank 3 conformer used as a query

\begin{tabular}{llll}
\hline Rank & ROCS hits & Shape tanimoto & Electrostatic tanimoto \\
\hline 1 & ZINC00022237 & 0.742 & -0.049 \\
2 & ZINC00018810 & 0.742 & 0.001 \\
3 & ZINC00014016 & 0.727 & -0.014 \\
4 & ZINC0006117 & 0.725 & 0.002 \\
5 & ZINC00014017 & 0.725 & -0.015 \\
\hline
\end{tabular}

Table 4: Docking scores of set 1 hits

\begin{tabular}{lllll}
\hline Rank & ROCS hits & Chemscore & Shapegauss & PLP \\
\hline 1 & ZINC00017019 & -7.612 & -267.2 & -29.25 \\
2 & ZINC00018810 & -8.365 & -329.4 & -36.22 \\
3 & ZINC0006117 & -9.464 & -273.7 & -18.92 \\
4 & ZINC00023049 & -3.401 & -238.4 & -31.85 \\
5 & ZINC0006708 & -4.591 & -257.7 & -16.59 \\
Query molecule & & 3.840 & -257.4 & -24.32 \\
\hline
\end{tabular}

molecule. We have randomly selected only Rank 1, 8 and 20 conformers for screening purpose, namely Set 13 conformers respectively and hits obtained using these conformers are named as Set 1-3 molecules, respectively. Totally 300 hits were obtained and each 100 hits for each set was ranked by ST value.

These ROCS hits were used as an input to EON in order to calculate electrostatic similarity between the query and ROCS hits. Spin $90^{\circ}$ command was used to maximize the electrostatic similarity. Finally it is 
ranked by Electrostatic Similarity (ET) value. Top five ranked molecules in each set were selected for further study (Table 1-3). The selection of hits based on ST and ET values elaborated in discussion. On the basis of higher structural and electrostatic similarity to query, five hits from Set 1 data set were docked into the active site of HIV-1 IN enzyme. Docking energy of five new molecules and Raltegravir (query) with HIV-1 IN are given in Table 4. Three scoring functions were used to compare the results. Figure 4 shows rank 5 molecule docked with HIV-IN active site.

\section{DISCUSSION}

This research has shown that it is possible to detect compounds with similar shape and electrostatics to a query molecule using ROCS and EON software. ROCS rapidly identify very similar compounds to Raltegravir such as Set 1 molecules. However, it fails when the electrostatics similarity in terms of ET value was compared, because of large similar shaped compounds does not show same activity. To solve this problem, other conformers of Raltegravir such as Set 2 and 3 were chosen for screening. But, in the case of Set 2 molecules, ST values were almost similar to Set 1 and ET values were very low than that of Set 1 compounds. Surprisingly ST values of Set 3 molecules were slightly higher than that of compounds in Sets 1 and 2. But ET values were very low than that of the other two sets, because of EON is conformer specific, that means different ET values we can obtain for different conformers of the same molecule.

To maximize the electrostatic similarity, spin $90^{\circ}$ command was used in EON for all the three sets. But there was no significant improvement in ET values of compounds in Sets 2 and 3. Among Set 1 compounds, fourth and fifth ranked compounds showed higher ET values. Between these two, Rank 5 compound was shown to have $50 \%$ similarity to query molecule.

Docking energies of Set 1 compounds were shown to have good binding affinity than query. Fifth ranked compound was selected as good inhibitor of HIV-1 IN enzyme, because it was showed higher binding affinity than query and $71 \%$ of structural similarity and $51 \%$ of electrostatic similarity to query. Hence the fifth ranked molecule ZINC0006708 has satisfied both shape and electrostatic similarity criteria.

\section{CONCLUSION}

ROCS, EON and FRED effectively identified one active inhibitor of HIV-1 IN among five compounds, which was similar to the query molecule and this study shows that ROCS, EON and FRED can play a vital role in drug discovery projects. ROCS is conformer specific, for example if we increase the number of conformers of query, then we get more number of hits. Increasing the number of molecules in the database is another way to get more number of hits. The selection of most active compounds from hits is very essential in virtual screening. In this study, EON and FRED played a vital role in compound selection. Synthesis and in vitro studies of hit molecules will support our study. Further study in this area will be required to design novel HIV1 IN inhibitors.

\section{REFERENCES}

Andrake, M.D. and A.M. Skalka, 1996. Retroviral integrase, putting the pieces together. J. Biol. Chem., $\quad$ 271: $19633 . \quad$ DOI: 10.1074/jbc.271.33.19633

Asante-Appiah, E. and A. M. Skalka, 1997. Molecular mechanisms in retrovirus DNA integration. Antivir. Res., 36: 139-156. DOI: 10.1016/S01663542(97)00046-6

Beese, L.S. and T.A. Steitz, 1991. Structural basis for the 3' -5' exonuclease activity of Escherichia coli DNA polymerase I: A two metal ion mechanism. EMBO. J., 10: 25-33. PMID: PMC452607

Bostrom, J., J.R. Greenwood and J. Gottfries, 2003. Assessing the performance of OMEGA with respect to retrieving bioactive conformations. J. Mol. Graph. Model., 21: 449-462. DOI: 10.1016/S1093-3263(02)00204-8

Bujacz, G. et al., 1995. High-resolution structure of the catalytic domain of avain sarcoma virus integrase. J. Mol. Biol., 253: 333-346. DOI: 10.1006/jmbi.1995.0556

Cai, M. et al., 1997. Solution structure of the Nterminal zinc binding domain of HIV-1 integrase. Nat. Struct. Mol. Biol., 4: 567-577. DOI: 10.1038/nsb/0797-567

Cocohoba, J. and B.J. Dong, 2008. The first HIV integrase inhibitor. Clin. Ther., 30: 1747-1765. DOI: 10.1016/j.clinthera.2008.10.012

Cozzi-Lepri, A., 2007. Evolution of drug resistance in HIV-infected patients remaining on a virologically failing combination antiretroviral therapy regimen. AIDS, 21: 721-732. $\quad$ DOI: 10.1097/QAD.0b013e3280141rdf

De Bethune, M.P. and K. Hertogs, 2006. Screening and selecting for optimized antiretroviral drugs: Rising to the challenge of drug resistance. Curr. Med. Res. Opin., 22: 2603-2612. DOI: 10.1185/030079906X154114 
Di Santo, R. et al., 2005. Design, synthesis and biological evaluation of heteroaryl diketohexenoic and diketobutanoic acids as HIV-1 integrase inhibitors endowed with antiretroviral activity. Farmaco, 60: 409-417. DOI: 10.1016/j.farmac.2005.03.008

Dyna, F. et al., 1994. Crystal structure of the catalytic domain of HIV-1 integrase: Similarity to other polynucleotidyl transferases. Science, 266: 1981-1986. DOI: 10.1126/science.7801124

Eijkelenboom, A.P. et al., 1995. The DNA binding domain of HIV-1 integrase has an SH3-like fold. Nat. Struct. Biol., 2: 807. DOI: 10.103/nsb0995-807

Ellison, V. and P.O. Brown, 1994. A stable complex between integrase and viral DNA ends mediates human immunodeficiency virus integration in vitro. Proc. Natl. Acad. Sci. USA., 91: 7316-7320. PMID: 8041787

Engelmen, A., A.B. Hickman and R. Craigie, 1994. The core and carboxy-terminal domains of the integrase protein of human immunodeficiency virus type 1 each contribute to nonspecific DNA binding. J. Virol., 68: 5911-5917. PMID: 8057470

Fontas, E. et al., 2004. Lipid profiles in HIV-infected patients receiving combination antiretroviral therapy: Are different antiretroviral drugs associated with different lipid profiles. Am. J. Infect. Dis., 189: 1056-1074. DOI: 10.1086/381783

FRED ver.2.2, 2006. Openeye Scientific Software, Inc. http://www.eyesopen.com

Gundersen, E. et al., 2005. Molecular-modeling based design, synthesis and activity of substituted piperidines as [gamma]-secretase inhibitors. Bioorg. Med. Chem. Lett., 15: 1891-1894. DOI: 10.1016/j.bmcl.2005.02.006

Halgren, T.A., 1996. Merck molecular force field I. Basis, form, scope, parameterization and performance of MMFF94. J. Comput. Chem., 17: 490-512. DOI: 10.1002/(SICI)1096987X(199604)17:5/6<490::AID-JCC1>3.0.CO;2-P

Hazuda, D.J. et al., 1997. Differential divalent cation requirement uncouple the assembly and catalytic reactions of human immunodeficiency virus type 1 integrase. J. Virol., 71: 7005-7011. PMID: 9261430

Hazuda, D.J. et al., 2000. Inhibitors of strand transfer that prevent integration of HIV-1 replication in cells. Science, 287: 646-650. DOI: 10.1126/science.287.5453.646

Hindmarsh, P. and J. Leis, 1999. Retroviral DNA integration. Microbiol. Mol. Biol. Rev., 63: 836-843. PMCID: PMC98978
John, J.I. and K.S. Brain, 2005. Zinc: A free database of commercially available compounds for virtual screening. J. Chem. Inform. Model., 45 (1): 177182. DOI: $10.1021 / \mathrm{ci049714.}$

Krajewski, K. et al., 2004. Synthesis and HIV-1 integrase inhibitory activity of dimeric and tetrameric analogs of indolicidin. Bioorg. Med. Chem. Lett., 14: 5595-5598. DOI: 10.1016/j.bmcl.2004.0.061

Laufer, R. et al., 2006. Preclinical pharmacokinetics metabolism of MK-0518, a potent HIV integrase inhibitor in phase III clinical trails. of the 16th AIDS International Conference, Aug. 13-18, Toronto, Abstract No. THPE0027

Lee, S.P. and M.K. Han, 1996. Zinc stimulates $\mathrm{Mg}^{2+}$ dependent 3'-processing activity of human immunodeficiency virus type 1 integrase in vitro. Biochemistry, 35: 3837-3844. DOI: 10.1021/bi952056p

Lee, S.P. et al., 1997. $\mathrm{Zn}^{2+}$ promotes the selfassociation of human immunodeficiency virus type 1 integrase in vitro. Biochemistry, 36: 173-180. DOI: 10.1021/bi961849o

Lodi, P. et al., 1995. Solution structure of the DNA binding domain of HIV-1 integrase. Biochemistry, 34: 9826. DOI: 10.1021/bi00031a002

Machouf, N., et al., 2006. Effects of drug resistance on viral load in patients failing antiretroviral therapy. J. Med. Virol., 78: 608-613. DOI: 10.1002/jmv.2052

Makhija, M.T. and V.M. Kulkarni, 2001. Molecular electrostatic potentials as input for the alignment of HIV-1 integrase inhibitors in 3D QSAR. J. Comput. Aided Mol. Des., 15: 961-978. DOI: 10.1023/A:1014888730876

Makhija, M.T. and V.M. Kulkarni, 2002. QSAR of HIV-1 integrase inhibitors by genetic function approximation method. Bioorg. Med. Chem., 10: $1483-1497 . \quad$ DOI: $10.1016 /$ S09680896(01)00415-1

Marchand, C. et al., 2006. Covalent binding of the natural antimicrobial peptide indolicidin to DNA a basic sites. Nucleic Acids Res., 34: 5157-5165. DOI: $10.1093 / \mathrm{nar} / \mathrm{gk} / 1667$

Markowitz, M. et al., 2006. Antiretroviral activity, pharmacokinetics and tolerability of MK-0518, a novel inhibitor of HIV-1 integrase, dosed as monotherapy for 10 days in treatment-native HIV1 infected individuals. J. Acquir. Immun. Defic. Syndr., 43: 509-515. DOI: 10.1097/QAI.0b013e31802b4956 
Morales-Ramirez, J. O. et al., 2005. Antiretroviral effect of MK-0518, a novel HIV-1 integrase inhibitor, in ART-naïve HIV-infected patients. Proceeding of the Program and Abstracts of the 10th European AIDS Conference, Nov. 17-20, European AIDS Clinical Society, Paris, Abstract LBPS1/6

Muchmore, S.W., A.J. Souers and I. AkritopoulouZanze, 2006. The use of three-dimensional shape and electrostatic similarity searching in the identification of Melanin-concentrating hormone receptor I antagonist. Chem. Biol. Drug Des., 67: 174-176. DOI: $10.1111 / \mathrm{j} .1747-$ 0285.2006.00341.x

OMEGA ver.2.1.0, 2006. Openeye Scientific Software, Inc. http://www.eyesopen.com

Potter, S.J. et al., 2006. Genetic analyses reveal structured HIV-1 populations in serially sampled T lymphocytes of patients receiving HAART. Virology, 348: 35-46. DOI: 10.1016/j.virol.2005.12.031

Potter, S. J., D. Dwyer and N. K. Saksena, 2003. Differential cellular distribution of HIV-1 drug resistance in vivo: Evidence for infection of CD8+T cells during HAART. Virology, 10: 339352. DOI: $10.1006 /$ viro.2002:1703

QuacPac ver.1.3, 2006. Openeye Scientific Software, Inc. http://www.eyesopen.com

Robinson Jr., N.E. et al., 1998. Anti-HIV-1 activity of indolicidin, an antimicrobial peptide from neutrophils. J. Leukoc. Biol., 63: 94-100. PMID: 9469478

ROCS ver.2.2 and EON ver.1.1, 2006. Openeye Scientific Software, Inc. http://www.eyesopen.com

Rush, T.S. et al., 2005. A shape-based 3-D scaffold hopping method and its application to a bacterial protein-protein interaction. J. Med. Chem., 48: 1489-1495. DOI: 10.1021/jm040163o

Schulz-Gasch, T. and M. Stahl, 2003. Binding site characteristics in structure-based virtual screening: Evaluation of current docking tools. J. Mol. Model., 9: 47-57. DOI: 10.1007/s00894-002-0112-y
Shiomi, K. et al., 2005. Fungal phenalenones inhibit HIV-1 integrase. J. Antibiot. (Tokyo), 58: 65-68. http://www.antibiotics.or.jp/journal/contents/vol58/abstract/58-1/ja580108.htm

Summa, V. et al., 2006. Discovery of MK-0518 a novel, potent and selective HIV integrase inhibitor in phase III clinical trails. Proceeding of the 16th AIDS International Conference, Aug. 13-18, Toronto, Abstract No. ThPe0020

Thomas, M. and L. Brandy, 1997. HIV integrase: A target for aids therapeutics. Trends Biotechnol., 15: 167-172. DOI: 10.1016/S0167-7799(97)01016-0

Tozzi, V. et al., 2006. Drug-class-wide resistance to antiretrovirals in HIV-infected patients failing therapy: Prevalence, risk factors and virological outcome. PMID: 16964822

Vink, C., R.A. Lutzke and R.H. Plasterk, 1994. Formation of stable complex between human immunodeficiency virus integrase protein and viral DNA. Nucleic Acid. Res., 22: 4103-4110. DOI: 10.1093/nar/22.20.4103

Woerner, A.M. and C.J. Marcus-Sekura, 1993. Characterization of a DNA binding domain in the C-terminal of HIV-1 integrase by deletion mutagenesis. Nucleic Acid. Res., 21: 3507-3511. PMID: 8346030

Wolfe, A.L. et al., 1996. The role of manganese in promoting multimerization and assembly of human immunodeficiency virus type 1 integrase as a catalytically active complex on immobilized long terminal repeat substrates. J. Virol., 70: 1424-1432. PMID: 8627659

Yuan, H. and A.L. Parrill, 2002. QSAR studies of HIV-1 integrase inhibition. Bioorg. Med. Chem., 10: 4169-4183. DOI: 10.1016/S09680896(02)00332-2

Yusa, K. and S. Harada, 2004. Acquisition of multi-PI (protease inhibitor) resistance in HIV-1 in vivo and in vitro. Curr. Pharm. Des., 10: 4055-4064. PMID: 15579087

Zheng, R., T.M. Jenkins and R. Craigie, 1996. Zinc folds the N-terminal domain of HIV-1 integrase, promotes multimerization and enhances catalytic activity. Proc. Natl. Acad. Sci., 93: 13659-13664. PMID: 8942990 